\title{
小麦矮秆突变体的鉴定及其突变性状的关联分析
}

\author{
贺军与 尹顺琼陈云琼熊静蕾 王卫斌 周鸿斌陈梅王梦玥 \\ 陈升位 ${ }^{*}$
}

云南农业大学农学与生物技术学院, 云南昆明 650201

摘 要: 矮秆突变体是小麦育种和株高遗传研究的重要基因资源。通过 ‘云麦 53’成熟种子的 EMS (Ethyl methyl sulfonate) 诱变及诱变植株连续自交, 获得了 33 个 $\mathrm{M}_{3}$ 代候选突变体。通过诱变亲本与 $\mathrm{M}_{2}$ 和 $\mathrm{M}_{3}$ 代候选植株的株高差异分析, 篮 选到 26 个矮秆突变体, 其株高变幅为(13.61 \pm 0.11$)(44.08 \pm 1.73) \mathrm{cm}$ 。基于 8 个矮秆基因的 12 个特异性标记检测发现, 26 个矮秆突变体至少携带 2 个矮秆基因标记位点。除株高外, 26 个矮秆突变体还携带穗长、小穗密度、节间数和平均节间 长 4 个不同突变性状。 26 个矮秆突变体可聚为 5 个亚类, 第 1 亚类的小穗数和小花数最少; 第 2 亚类的株高最矮, 穗长 和平均节间长最短, 小穗密度最高; 第 3 亚类突变体的节间数最少。株高与平均节间长和节间数呈极显著相关, 偏相关 系数分别为 $0.94 、 0.58$, 但与穗长、小穗数、小花数和小穗密度 4 个性状无相关性。 26 个矮秆突变体的株高与平均节间 长和节间数关联遗传, 携带不同的突变基因组合, 可用于小麦矮化育种, 以及株高、穗长和小穗密度等性状的遗传机制 研究。

关键词: 小麦; 突变体; 矮秆基因; 分子标记; 遗传关联

\section{Identification of wheat dwarf mutants and analysis on association between the mutant traits of the dwarf plants}

HE Jun-Yu, YIN Shun-Qiong, CHEN Yun-Qiong, XIONG Jing-Lei, WANG Wei-Bin, ZHOU Hong-Bin, CHEN Mei, WANG Meng-Yue, and CHEN Sheng-Wei*

Collage of Agronomy and Biotechnology, Yunnan Agricultural University, Kunming 650201, Yunnan, China

\begin{abstract}
Dwarf mutant is an important gene resource in wheat breeding and plant height genetic research. In this paper, EMS (ethyl methyl sulfonate) was used to mutate the mature seeds of 'Yunmai 53', and 33 candidate dwarf mutants of $\mathrm{M}_{3}$ generation were finally obtained by self-bred. Twenty-six dwarf mutants were selected by analyzing differences between the mutant parent in two years and that of $\mathrm{M}_{2}$ and $\mathrm{M}_{3}$ generation candidate plants, and the variation range of their plant height was from (13.61 \pm 0.11$)$ $\mathrm{cm}$ to $(44.08 \pm 1.73) \mathrm{cm}$. Twenty-six dwarf mutants with at least 2 mutant sites were verified based on 12 specific markers of 8 dwarf genes. In addition to plant height, 26 dwarf mutants also carried four mutational traits, spike length, spikelet density, internode number, and average internode length. The 26 dwarf mutants could be clustered into 5 subgroups. Among them, the first subgroup was the least in spikelets and florets, the second subgroup was the shortest in plant height, spike length and average internode length, and the highest spikelet density, while the third subgroup was the least in internode number. Plant height was significantly correlated with average internode length and internode number with partial correlation coefficients of 0.94 and 0.58 , respectively, but not correlated with spike length, spikelet number and spikelet density. The plant height was genetically associated with internode length and internode number in 26 dwarf mutants. The mutants carried different combinations of the mutant gene, and can be useful in wheat dwarf breeding and the studies on genetic mechanism of the traits, such as plant height, spike length and spikelet density.
\end{abstract}

Keywords: wheat; mutant; dwarf genes; molecular marker; genetic association

\footnotetext{
本研究由国家自然科学基金项目(31660434, 32060457)资助。

This study was supported by the National Natural Science Foundation of China (31660434, 32060457).

*通信作者(Corresponding author): 陈升位, E-mail: ynkmcsw@126.com

第一作者联系方式: E-mail: etfiee_coisini@126.com

Received (收稿日期): 2020-08-16; Accepted (接受日期): 2020-11-13; Published online (网络出版日期): 2020-12-15.

URL: https://kns.cnki.net/kcms/detail/11.1809.S.20201214.1749.006.html
} 
自“绿色革命”以来，全球种植的小麦品种中有 $90 \%$ 含有矮秆和半矮秆基因 ${ }^{[1-5]}$ 。迄今为止, 已报道的矮秆和 半矮秆基因超过 25 个, 但可用于小麦育种的矮秆基因局

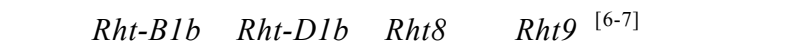
$R h t-B 1 b 、 R h t-D 1 b$ 和 $R h t 8$ 在降低小麦株高时对其他性状 无显著不良影响, 且 $R h t-D 1 b 、 R h t 8$ 和 $R h t 9$ 可显著增加穗 粒数 ${ }^{[8-12]}$ 。李杏普等 ${ }^{[13]}$ 发现 $R h t 10$ 和 $R h t 12$ 的降秆作用大 于 $R h t 8$, 但其株系的地上部分生物量低于 $R h t 8$ 株系。王 清海等 ${ }^{[14]}$ 研究发现 Rht14、Rht16 和 Rht18 的降秆效应分 别为 $47.3 \% 、 39.9 \%$ 和 $36.0 \%$ 。携带 Rht $14 、 R h t 16$ 和 Rht 18 的矮秆株系的小穗小花数变化不大, 但穗长变长。Rht5 在降低小麦株高的同时会减少每穗小穗数和穗粒数 ${ }^{[15]}$ 。 前人研究表明, 大多数小麦推广品种携带的矮秆基因为

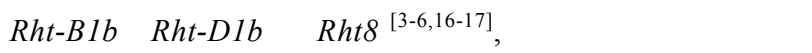
本不足, 育成品种遗传背景狭窄。创制新突变体是解决该 问题的有效策略。本文采用甲基磺酸乙酯(Ethyl methyl sulfonate, EMS)诱变“云麦 53’ (国审品种)成熟种子, 通过 诱变植株的连续 3 年套袋自交成功获得了一批株高、小穗 密度和小花数等性状不同的候选突变体。基于连续 2 年的 田间观察和常见矮秆基因的 12 个特异性分子标记检测鉴 定了 26 株矮秆突变株, 分析了 26 个突变体的株高差异及 其与穗长、小穗数和小穗密度等农艺性状的遗传关联性, 评估了矮秆突变体的育种价值。

\section{1 材料与方法}

\section{1 试验材料}

诱变亲本“云麦 53 ’由云南省农业科学研究院粮食作 物研究所提供, 突变体由课题组创制。所有材料由课题组 繁育并保存。

\section{2 试验材料的种植及取样}

1.2.1 材料诱变和繁育 将 15,000 粒‘云麦 53 ’种子置
于三角瓶中, 用 $\mathrm{pH} 8.0$ 的 $0.05 \mathrm{~mol} \mathrm{~L}^{-1}$ 磷酸缓冲液(PBS) 震荡浸泡 $4 \mathrm{~h}$ ，再用 $0.6 \%$ EMS 室温震荡浸泡 $16 \mathrm{~h}$, 然后用 自来水冲洗 $4 \mathrm{~h}$ 。诱变种子及其后代均播种于云南农业大 学昆明市盘龙区试验教学农场。分别套袋自交 $M_{1} 、 M_{2}$ 和 $\mathrm{M}_{3}$ 候选突变材料, 按单株收获种子。

\subsection{2 农艺性状调查按随机排列种植试验材料, 每} 个材料种植 1 行, 5 行后种植 1 行诱变亲本。行距 $20 \mathrm{~cm}$, 株 距 $5 \mathrm{~cm}, 3$ 次重复。其他田间管理措施与常规大田管理一 致。于 2018-2019 年度和 2019-2020 年度分别在开花期 调查穗长、小穗数和小花数, 待种子成熟后调查株高、平 均节间长和节间数。 $\mathrm{M}_{2}$ 代调查单株性状, $\mathrm{M}_{3}$ 株系随机调 查 5 株, 以 5 株均值代表株系性状的表型值。按下列公式 计算小穗密度、平均节间长。

小穗密度 $=$ 单株小穗数 $/$ 单株穗长

平均节间长 $=$ 单株节间长 $/$ 单株节间数

1.2.3 矮秆基因的分子标记检测 于拔节期剪取 $\mathrm{M}_{2}$ 单 株及其诱变亲本的幼嫩叶片, 置于 $-80^{\circ} \mathrm{C}$ 保存备用。采用 CTAB 法提取基因组 DNA。采用 Ellis 等 ${ }^{[18-19]}$ 报道的 BF-WR1、BF-MR1、BF-WR2、BF-MR2、WMC317、 BARC102、BARC151、WMC410 和 WMS577, 以及 $\mathrm{XBARC}^{[20]} 、 \mathrm{Xgwm} 261^{[20]}$ 和 WMC503 ${ }^{[21]}$ 特异性标记检测 突变体携带的矮秆基因(表 1), 其中 BF-WR1 和 BF2-WR2 为野生型(株高正常)基因标记, 其余标记为突变型(矮秆) 基因标记。由上海生工生物技术有限公司合成所有引物。 参照前人报道的 PCR 扩增条件扩增各标记, 采用 $8 \%$ 聚丙 烯酰胺凝胶电泳检测 PCR 扩增产物。上述标记均重复检 测 3 次, 3 次检测结果一致。

1.2.4 农艺性状的关联分析 基于 SPSS 25.0 软件的 $t$ 测验、聚类、方差、多重比较和相关分析模块默认参数, 分 析突变体株高差异及其与穗长、小穗数和小穗密度等性状 的遗传关联性。显著和极显著水平分别为 $5 \%$ 和 $1 \%$ 。

表 1 矮秆基因及其特异性标记的引物序列和片段长度

Table 1 Specific molecular markers of dwarf genes and the fragment length and primers sequence of the markers

\begin{tabular}{|c|c|c|c|c|}
\hline $\begin{array}{l}\text { 基因 } \\
\text { Gene }\end{array}$ & $\begin{array}{c}\text { 标记 } \\
\text { Marker }\end{array}$ & $\begin{array}{c}\text { 正向引物 } \\
\text { Forward sequence } \\
\left(5^{\prime}-3^{\prime}\right)\end{array}$ & $\begin{array}{c}\text { 反向引物 } \\
\text { Reverse sequence } \\
\left(5^{\prime}-3^{\prime}\right)\end{array}$ & $\begin{array}{l}\text { 片段长度 } \\
\text { Product size } \\
\text { (bp) }\end{array}$ \\
\hline Rht-Bla & BF-WR1 & GGTAGGGAGGCGAGAGGCGAG & CATCCCCATGGCCATCTCGAGCTG & 237 \\
\hline$R h t-B 1 b$ & BF-MR1 & GGTAGGGAGGCGAGAGGCGAG & CATCCCCATGGCCATCTCGAGCTA & 237 \\
\hline Rht-Dla & BF2-WR2 & GGCAAGCAAAAGCTTCGCG & GGCCATCTCGAGCTGCAC & 264 \\
\hline Rht-D1b & BF-MR2 & CGCGCAATTATTGGCCAGAGATAG & CCCCATGGCCATCTCGAGCTGCTA & 254 \\
\hline Rht4 & WMC317 & TGCTAGCAATGCTCCGGGTAAC & TCACGAAACCTTTTCCTCCTCC & 170 \\
\hline Rht 5 & BARC102 & GGAGAGGACCTGCTAAAATCGAAGACA & GCGTTTACGGATCAGTGTTGGAGA & 200 \\
\hline Rht 8 & Xgwm261 & CTCCCTGTACGCCTAAGGC & CTCGCGCTACTAGCCATTG & 192 \\
\hline Rht 8 & WMC503 & GCAATAGTTCCCGCAAGAAAAG & ATCAACTACCTCCAGATCCCGT & 225 \\
\hline Rht9 & BARC151 & TGAGGAAAATGTCTCTATAGCATCC & CGCATAAACACCTTCGCTCTTCCACTC & 220 \\
\hline Rht 12 & WMC410 & GGACTTGAAAGGAAGCTTGTGA & CATGGATGGCATGCAGTGT & 114 \\
\hline Rht13 & WMS577 & ATGGCATAATTTGGTGAAATTG & TGTTTCAAGCCCAACTTCTATT & 130 \\
\hline Rht $14 / 16 / 18$ & XBARC3 & TTCCCTGTGTCTTTCTAATTTTTTTT & GCGAACTCCCGAACATTTTTAT & 210 \\
\hline
\end{tabular}




\section{2 结果与分析}

\section{1 候选矮秆突变体的农艺性状差异分析}

本研究采用 $t$ 测验检测了 ‘云麦 53' 与 33 个候选材料的 株高 $\left(\mathrm{M}_{2}\right.$ 单株表型值, $\mathrm{M}_{3}$ 株系均值)差异(表 2)。结果表明, mutant 2、mutant 4、mutant 5 等 26 个材料与“云麦 53 ’的株 高存在显著或极显著差异, 为矮秆突变体。在 26 个矮秆突 变体中 mutant 16、 mutant 23、 mutant 25 和 mutant 26 的株 高值低于 $20 \mathrm{~cm}$, mutant 18 、 mutant 19 和 mutant 20 的株高 值大于 $40 \mathrm{~cm}$, 其余 19 个突变体的株高值介于 $20 \sim 40 \mathrm{~cm}$
之间(图 1)。与“云麦 53’相比, mutant 4、mutant 5 和 mutant 7 等 18 个突变体的穗长显著或极显著缩短; mutant 4 、 mutant 5 和 mutant 7 等 11 个突变体的小穗密度显著或极显著增加; mutant 18 的小花数显著减少; mutant 5、mutant 11 、 mutant 14 等 13 个突变体的平均节间长显著或极显著缩短; mutant 5 、 mutant 10 和 mutant 14 等 6 个突变体的节间数显著或极显著 减少(表 2 和图 2)。mutant 7、mutant 12、mutant 24、mutant 28 和 mutant 29 的节间数在 $\mathrm{M}_{2}$ 和 $\mathrm{M}_{3}$ 代中未发生分离, 均低于 '云麦 53'的节间数, 但因方差为 0 、未做 $t$ 测验。26 个矮秆 突变体携带株高、穗长和小穗密度等多个突变性状。

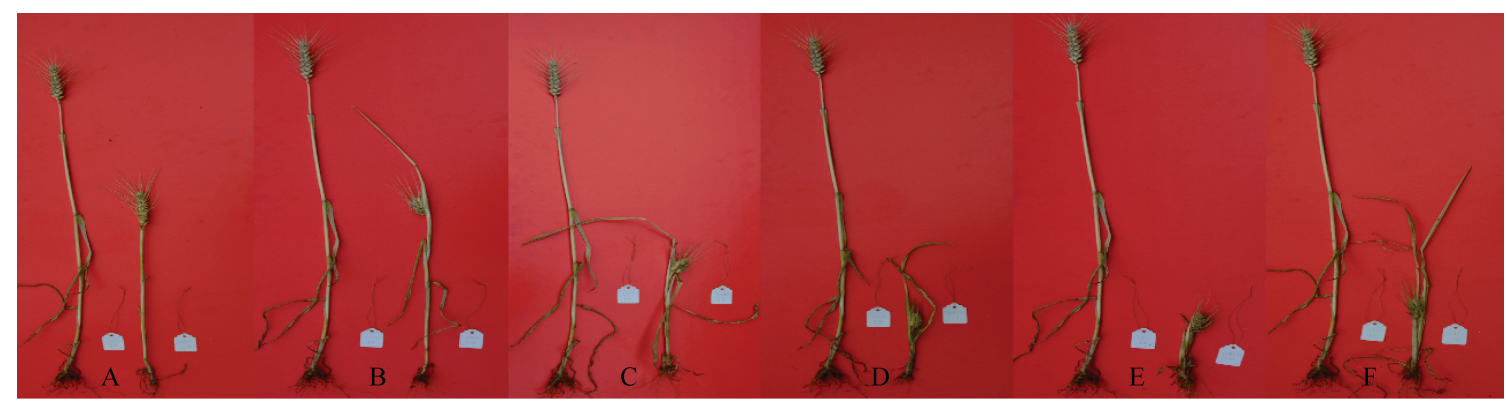

图 1 '云麦 53' 与部分矮秆突变体的株高差异

Fig. 1 Differences of plant height between 'Yunmai 53' and other dwarf mutants

A：突变体 $31 ; \mathrm{B}$ ：突变体 $17 ; \mathrm{C}$ ：突变体 $10 ; \mathrm{D}$ ：突变体 $16 ; \mathrm{E}$ ：突变体 $23 ; \mathrm{F}$ ：突变体 25 。

A: mutant 31; B: mutant 17; C: mutant 10; D: mutant 16; E: mutant 23; F: mutant 25.

表 2 '云麦 53' 及候选突变体的 7 个农艺性状均值及其显著性 $(t$ 测验)

Table 2 Mean values of 7 agronomic traits of 'Yunmai 53' and candidate mutants, and the difference between the mean values $(t$-test)

\begin{tabular}{|c|c|c|c|c|c|c|c|}
\hline $\begin{array}{c}\text { 材料 } \\
\text { Material }\end{array}$ & $\begin{array}{c}\text { 株高 } \\
\text { Plant height } \\
\text { (cm) }\end{array}$ & $\begin{array}{c}\text { 穗长 } \\
\text { Spike length } \\
(\mathrm{cm})\end{array}$ & $\begin{array}{c}\text { 每穗小穗数 } \\
\text { Spikelets number } \\
\text { per spike }\end{array}$ & $\begin{array}{c}\text { 小穗密度 } \\
\text { Spikelet } \\
\text { density }\end{array}$ & $\begin{array}{c}\text { 小花数 } \\
\text { Number of } \\
\text { florets }\end{array}$ & $\begin{array}{c}\text { 平均节间长 } \\
\text { Average internode } \\
\text { length }(\mathrm{cm})\end{array}$ & $\begin{array}{c}\text { 节间数 } \\
\text { Number of } \\
\text { internodes }\end{array}$ \\
\hline 云麦 53 Yunmai 53 & $74.89 \pm 4.03$ & $10.30 \pm 0.31$ & $19.25 \pm 1.21$ & $1.88 \pm 0.18$ & $59.35 \pm 5.23$ & $11.10 \pm 1.04$ & $6.00 \pm 0.00$ \\
\hline mutant 1 & $56.95 \pm 6.95$ & $10.49 \pm 1.52$ & $20.50 \pm 3.50$ & $2.05 \pm 0.63$ & $60.34 \pm 9.34$ & $7.50 \pm 3.33$ & $5.00 \pm 0.00$ \\
\hline mutant 2 & $39.52 \pm 2.02^{*}$ & $8.40 \pm 1.10$ & $17.30 \pm 3.30$ & $2.15 \pm 0.68$ & $58.20 \pm 16.20$ & $7.98 \pm 0.78$ & $4.50 \pm 0.50$ \\
\hline mutant 3 & $63.99 \pm 2.51$ & $6.11 \pm 0.59^{*}$ & $17.10 \pm 1.10$ & $2.84 \pm 0.46$ & $51.70 \pm 3.70$ & $11.23 \pm 0.96$ & $5.40 \pm 0.60$ \\
\hline mutant 4 & $37.24 \pm 0.44^{*}$ & $4.44 \pm 0.34^{* *}$ & $19.30 \pm 1.30$ & $4.35 \pm 0.04^{* *}$ & $55.00 \pm 1.00$ & $7.21 \pm 0.61$ & $4.50 \pm 0.50$ \\
\hline mutant 5 & $21.94 \pm 3.66^{*}$ & $3.72 \pm 0.02^{* *}$ & $20.00 \pm 1.00$ & $5.37 \pm 0.24^{* *}$ & $58.30 \pm 1.30$ & $4.88 \pm 0.62^{*}$ & $3.80 \pm 0.20^{* *}$ \\
\hline mutant 6 & $39.85 \pm 4.39^{*}$ & $6.01 \pm 0.45^{*}$ & $19.10 \pm 2.10$ & $3.73 \pm 1.27$ & $55.50 \pm 4.50$ & $6.87 \pm 0.44$ & $4.50 \pm 0.50$ \\
\hline mutant 7 & $33.89 \pm 2.89^{*}$ & $4.71 \pm 0.41^{* *}$ & $21.00 \pm 2.00$ & $4.46 \pm 0.04^{* *}$ & $63.40 \pm 6.40$ & $7.22 \pm 0.39$ & $4.00 \pm 0.00$ \\
\hline mutant 8 & $34.92 \pm 2.58^{*}$ & $4.40 \pm 0.10^{* *}$ & $22.50 \pm 0.50$ & $5.12 \pm 0.01^{* *}$ & $67.50 \pm 1.50$ & $7.16 \pm 0.44$ & $4.40 \pm 0.60$ \\
\hline mutant 9 & $31.81 \pm 6.79^{*}$ & $4.18 \pm 0.58^{*}$ & $21.30 \pm 2.30$ & $5.12 \pm 0.16^{* *}$ & $61.20 \pm 4.20$ & $6.20 \pm 0.95$ & $4.40 \pm 0.60$ \\
\hline mutant 10 & $25.55 \pm 7.85^{*}$ & $4.24 \pm 0.35^{* *}$ & $19.40 \pm 1.40$ & $4.62 \pm 0.71$ & $54.70 \pm 0.70$ & $5.44 \pm 1.77$ & $3.90 \pm 0.10^{* *}$ \\
\hline mutant 11 & $29.78 \pm 1.92^{*}$ & $8.20 \pm 0.70$ & $16.10 \pm 0.90$ & $1.97 \pm 0.06$ & $48.00 \pm 3.00$ & $5.49 \pm 0.24^{*}$ & $4.50 \pm 0.50$ \\
\hline mutant 12 & $38.79 \pm 2.61^{*}$ & $4.63 \pm 0.13^{* *}$ & $19.30 \pm 2.30$ & $4.16 \pm 0.38^{*}$ & $54.40 \pm 3.40$ & $7.30 \pm 0.08$ & $5.00 \pm 0.00$ \\
\hline mutant 13 & $38.32 \pm 8.48$ & $3.98 \pm 0.38^{* *}$ & $15.90 \pm 1.90$ & $3.98 \pm 0.10^{* *}$ & $44.90 \pm 2.90$ & $6.65 \pm 0.85$ & $4.50 \pm 0.50$ \\
\hline mutant 14 & $33.18 \pm 6.02^{*}$ & $4.36 \pm 0.24^{* *}$ & $18.80 \pm 0.80$ & $4.34 \pm 0.43^{*}$ & $53.00 \pm 1.00$ & $5.95 \pm 0.03^{*}$ & $3.90 \pm 0.01^{* *}$ \\
\hline mutant 15 & $30.94 \pm 2.26^{*}$ & $4.00 \pm 0.50^{* *}$ & $17.90 \pm 0.10$ & $4.55 \pm 0.59^{*}$ & $49.50 \pm 4.50$ & $5.68 \pm 0.24^{*}$ & $4.80 \pm 0.20^{*}$ \\
\hline mutant 16 & $13.61 \pm 0.11^{* *}$ & $3.39 \pm 0.11^{* *}$ & $18.40 \pm 1.40$ & $5.45 \pm 0.59^{*}$ & $45.70 \pm 5.30$ & $2.26 \pm 0.63^{*}$ & $4.80 \pm 1.20$ \\
\hline mutant 17 & $32.62 \pm 4.98^{*}$ & $3.96 \pm 0.26^{* *}$ & $17.50 \pm 0.50$ & $4.43 \pm 0.16^{* *}$ & $49.10 \pm 1.90$ & $6.16 \pm 0.62$ & $4.50 \pm 0.50$ \\
\hline mutant 18 & $44.08 \pm 1.73^{*}$ & $7.08 \pm 0.48^{*}$ & $13.50 \pm 2.50$ & $1.90 \pm 0.23$ & $33.25 \pm 0.25^{*}$ & $7.87 \pm 0.97$ & $5.50 \pm 0.50$ \\
\hline mutant 19 & $42.41 \pm 12.19$ & $10.58 \pm 1.52$ & $21.40 \pm 2.40$ & $2.10 \pm 0.53$ & $69.10 \pm 12.10$ & $6.10 \pm 1.01$ & $5.30 \pm 0.70$ \\
\hline mutant 20 & $42.18 \pm 6.58$ & $7.97 \pm 0.63$ & $15.30 \pm 0.30$ & $1.94 \pm 0.20$ & $43.10 \pm 1.90$ & $7.31 \pm 1.89$ & $4.80 \pm 0.20^{*}$ \\
\hline mutant 21 & $31.26 \pm 4.44^{*}$ & $4.02 \pm 0.48^{* *}$ & $15.60 \pm 1.60$ & $3.99 \pm 0.88$ & $40.40 \pm 1.60$ & $7.04 \pm 0.79$ & $3.80 \pm 0.20^{* *}$ \\
\hline mutant 22 & $52.28 \pm 5.48$ & $8.45 \pm 0.95$ & $19.50 \pm 0.50$ & $2.34 \pm 0.33$ & $56.30 \pm 0.70$ & $8.44 \pm 0.94$ & $5.50 \pm 0.50$ \\
\hline
\end{tabular}




\begin{tabular}{|c|c|c|c|c|c|c|c|}
\hline $\begin{array}{c}\text { 材料 } \\
\text { Material }\end{array}$ & $\begin{array}{c}\text { 株高 } \\
\text { Plant height } \\
(\mathrm{cm}) \\
\end{array}$ & $\begin{array}{c}\text { 穗长 } \\
\text { Spike length } \\
(\mathrm{cm}) \\
\end{array}$ & $\begin{array}{c}\text { 每穗小穗数 } \\
\text { Spikelets number } \\
\text { per spike } \\
\end{array}$ & $\begin{array}{c}\text { 小穗密度 } \\
\text { Spikelet } \\
\text { density }\end{array}$ & $\begin{array}{c}\text { 小花数 } \\
\text { Number of } \\
\text { florets }\end{array}$ & $\begin{array}{c}\text { 平均节间长 } \\
\text { Average internode } \\
\text { length }(\mathrm{cm})\end{array}$ & $\begin{array}{c}\text { 节间数 } \\
\text { Number of } \\
\text { internodes }\end{array}$ \\
\hline mutant 23 & $15.80 \pm 0.60^{* *}$ & $3.70 \pm 0.35^{* *}$ & $19.80 \pm 1.80$ & $5.28 \pm 0.01^{*}$ & $58.80 \pm 4.80$ & $2.29 \pm 0.41^{*}$ & $4.40 \pm 0.60$ \\
\hline mutant 24 & $30.07 \pm 4.43^{*}$ & $6.76 \pm 1.04$ & $16.90 \pm 2.90$ & $2.63 \pm 0.84$ & $49.70 \pm 7.70$ & $5.26 \pm 0.05^{*}$ & $5.00 \pm 0.00$ \\
\hline mutant 25 & $18.16 \pm 4.44^{*}$ & $3.77 \pm 0.17^{* *}$ & $20.20 \pm 0.20$ & $5.37 \pm 0.19^{* *}$ & $58.80 \pm 1.20$ & $3.93 \pm 0.93^{*}$ & $4.50 \pm 0.50$ \\
\hline mutant 26 & $19.75 \pm 1.35^{* *}$ & $4.60 \pm 1.00^{*}$ & $18.60 \pm 1.60$ & $4.17 \pm 0.56$ & $53.20 \pm 2.20$ & $4.16 \pm 0.46^{*}$ & $3.80 \pm 0.20^{* *}$ \\
\hline mutant 27 & $34.54 \pm 4.74^{*}$ & $7.49 \pm 0.89$ & $14.80 \pm 3.80$ & $1.95 \pm 0.28$ & $50.00 \pm 17.00$ & $7.13 \pm 1.40$ & $4.50 \pm 0.50$ \\
\hline mutant 28 & $33.76 \pm 10.16$ & $8.86 \pm 0.16$ & $15.90 \pm 1.90$ & $1.79 \pm 0.18$ & $53.90 \pm 11.90$ & $6.59 \pm 3.49$ & $5.00 \pm 0.00$ \\
\hline mutant 29 & $35.01 \pm 4.79^{*}$ & $8.20 \pm 0.40$ & $16.40 \pm 0.40$ & $2.01 \pm 0.15$ & $49.30 \pm 1.30$ & $6.18 \pm 0.02^{*}$ & $5.00 \pm 0.00$ \\
\hline mutant 30 & $30.09 \pm 6.01^{*}$ & $8.57 \pm 1.13$ & $16.10 \pm 1.10$ & $1.93 \pm 0.38$ & $48.60 \pm 3.60$ & $5.92 \pm 0.00^{*}$ & $4.30 \pm 0.70$ \\
\hline mutant 31 & $33.96 \pm 0.36^{*}$ & $8.70 \pm 1.00$ & $17.20 \pm 0.20$ & $2.01 \pm 0.26$ & $49.20 \pm 1.80$ & $5.60 \pm 0.94$ & $4.50 \pm 0.50$ \\
\hline mutant 32 & $31.50 \pm 0.70^{* *}$ & $8.02 \pm 0.28^{*}$ & $16.50 \pm 1.50$ & $2.07 \pm 0.26$ & $46.30 \pm 1.30$ & $5.11 \pm 0.53^{*}$ & $4.50 \pm 0.50$ \\
\hline mutant 33 & $24.02 \pm 3.38^{*}$ & $10.04 \pm 1.94$ & $16.63 \pm 1.63$ & $1.69 \pm 0.17$ & $47.75 \pm 2.75$ & $3.95 \pm 0.12^{*}$ & $5.00 \pm 1.00$ \\
\hline
\end{tabular}

"和 ${ }^{* *}$ 分别代表 0.05 和 0.01 显著水平。 ${ }^{*}$ and ${ }^{* *}$ represent significant differences at the 0.05 and 0.01 probability levels, respectively.

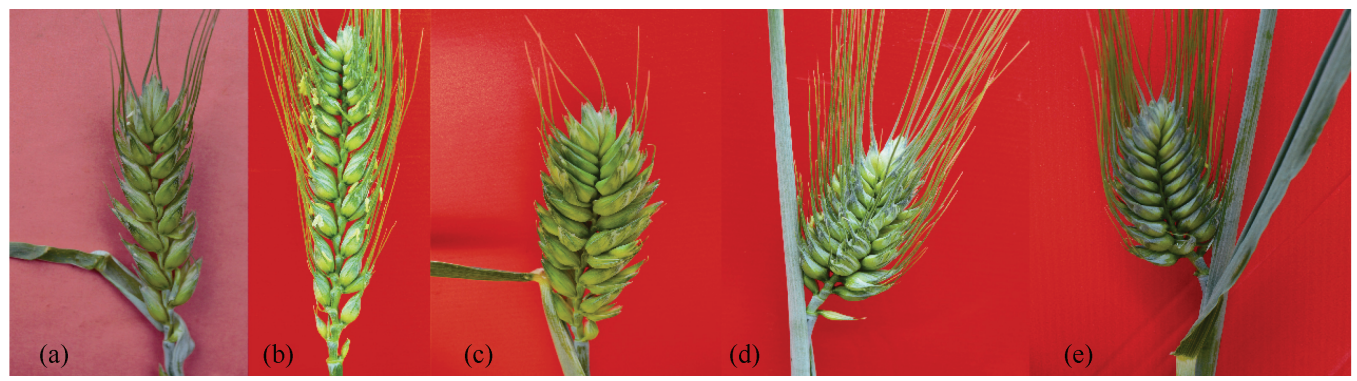

图 2 部分矮秆突变体及其诱变亲本的穗部形态特征

Fig. 2 Spike characteristic of some dwarf mutants and their parents

(a): ‘云麦 53 ’; (b), (c), (d)和(e): 候选矮秆突变体。

(a): 'Yunmai 53'; (b), (c), (d), and (e): candidate dwarf mutants.

\section{2 候选矮秆突变体的分子标记检测}

为了进一步验证 26 个矮秆材料, 本文采用前人报道的 矮秆基因的 12 个特异性标记检测了 33 个候选材料及其诱变 亲本。结果表明, 在“云麦 53’中未检测到 BF-MR1 (Rht-Blb)、 BF2-WR2 (Rht-D1a)、BARC102 (Rht5)和 WMS577 (Rht13) 标记, 但检测到其他 9 个标记。与“云麦 53 ’相比, mutant 1 等 32 个候选突变体的 BARC151 (Rht 9 )标记, mutant 2 等 5 个候
选突变体的 Xgwm261 (Rht8)、WMC503 (Rht8)标记均具有差 异, 表明其 $R h t 9$ 和 $R h t 8$ 基因发生了突变。在 33 个候选突变 体中, mutant 3 与“云麦 53’的共有扩增标记较多 (7 个), mu$\operatorname{tant} 11$ 与“云麦 53’仅有 2 个共同扩增标记(表 3 和图 3), mutant 1、mutant 2、 mutant 3、 mutant 27 和 mutant 28 的 Xgwm261 (Rht8)、WMC503 (Rht8)和 BARC151 (Rht9)均发生 变异, 其中 BARC151 (Rht 9 )标记位点的突变频率最高(表 3$)$ 。

表 3 '云麦 53'和候选突变体中检测到的矮秆基因

Table 3 Dwarf genes detected in the genome of 'Yunmai 53' and candidate mutants

\begin{tabular}{|c|c|}
\hline 材料 & 检测到的基因 \\
\hline Material & Genes detected by 13 markers \\
\hline 云麦 53 Yunmai 53 & Rht-B1a, Rht-D1b, Rht4, Rht8, Rht9, Rht12, Rht14, Rht16, Rht18 \\
\hline mutant 1 & Rht-B1a, Rht-Dla, Rht4, Rht5, Rht $8^{\#}, R h t 9^{\#}, R h t 13, R h t 14, R h t 16, R h t 18$ \\
\hline mutant 2 & 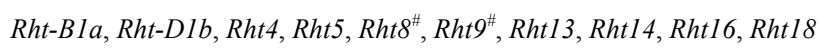 \\
\hline mutant 3 & Rht-B1a, Rht-D1b, Rht4, Rht8 ${ }^{\#}, R h t 9^{\#}, R h t 12, R h t 14, R h t 16, R h t 18$ \\
\hline mutant 10 & 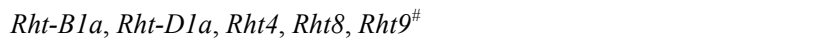 \\
\hline mutant 11 & Rht-B1a, Rht-Dla, Rht5, Rht12 \\
\hline mutant 19 & Rht-B1a, Rht-D1a, Rht4, Rht5, Rht8, Rht9 ${ }^{\#}$, Rht14, Rht16, Rht18 \\
\hline mutant 26 & Rht-B1a, Rht-D1b, Rht4, Rht8, Rht9, Rht13 \\
\hline mutant $27 \&$ mutant 28 & 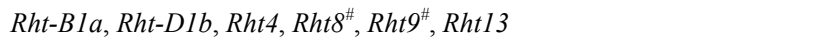 \\
\hline Other mutants & Rht-B1a, Rht-Dla, Rht4, Rht5, Rht8, Rht $9^{\#}$ \\
\hline
\end{tabular}

\footnotetext{
“\#”:多态性标记; Rht8 两个标记(Xgwm261、WMC503)在试验材料间的多态性一致。

“\#”: polymorphism markers in the fragment. The polymorphism of Xgwm261 and WMC503 of Rht8 show consistent among the experimental materials.
} 


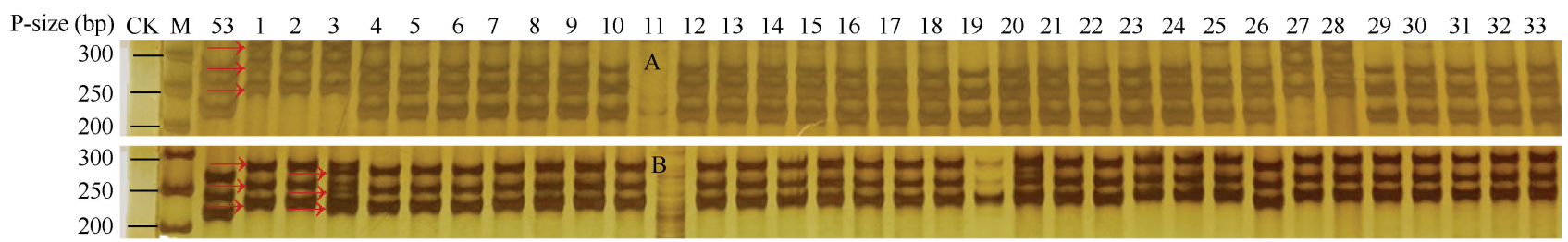

图 3 '云麦 53 ' 和候选突变体的部分特异性扩增标记

Fig. 3 Specific markers amplified from the genomes of 'Yunmai 53' and candidate mutants

CK：对照; M: marker; 53：云麦 53; 1 33：突变体 1 突变体 33; A 和 B 分别是‘云麦 53’和候选突变体中扩增的 WMC503 和 BARC151 标记; 红色箭: 差异片段。

CK: control; M: marker; 53: Yunmai 53; 1-33: mutant 1-mutant 33; A-B were WMC503 and BARC151 markers amplified in 'Yunmai 53' and candidate mutants, respectively. The red arrows indicate differential fragments.

\section{3 基于 7 个性状的矮秆突变体聚类分析}

基于株高、穗长和小穗密度等 7 个性状的聚类分析结 果表明, 所有材料可聚为 2 个大类, 第 1 大类包括诱变亲本, 第 2 大类包括所有突变体(图 4)。第 2 大类可分为 5 个亚类, 其中 mutant 18 和 mutant 16 分别聚为第 1 、2 亚类, mutant 5 、 mutant 10 和 mutant 23 等 5 个突变体为第 3 亚类, mutant 2 、 mutant 4 和 mutant 6 等 7 个突变体为第 4 亚类, 其

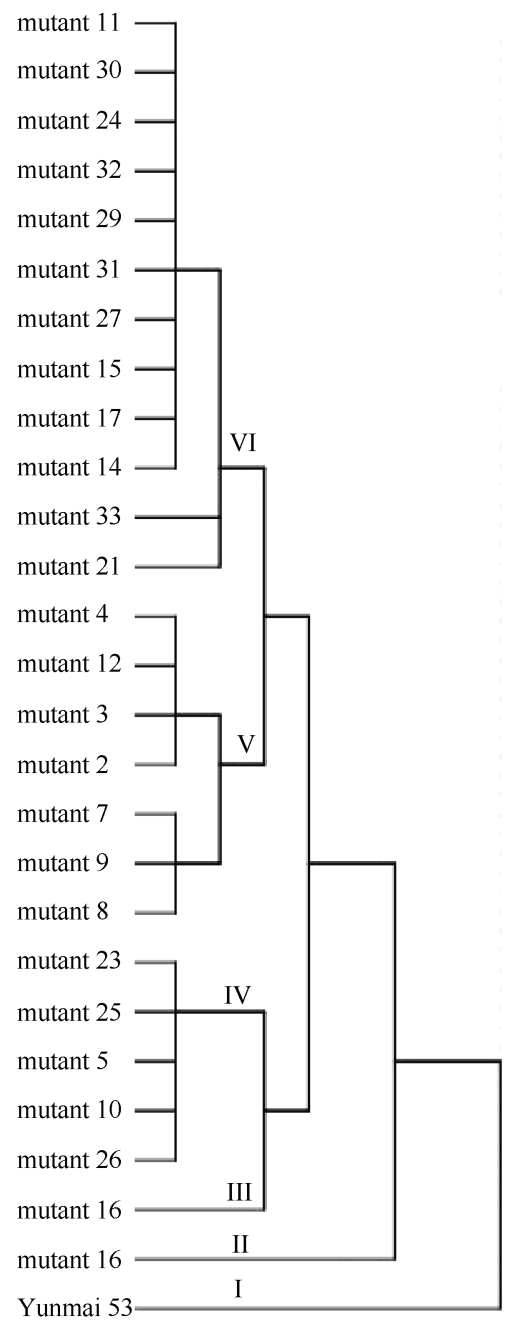

图 4 基于 7 个农艺性状的 26 个突变体和诱变亲本聚类结果 Fig. 4 Cluster result of 26 mutants and their parent based on seven agronomic traits
余 12 个突变体为第 5 亚类(图 4)。与“云麦 53 ’相比, 5 个 亚类的突变体均表现植株矮化、穗长和平均节间长缩短, 但其余 4 个性状出现了分化。其中第 1 亚类突变体的小穗 数和小花数极显著减少, 但小穗密度和节间数没有差异; 第2 4亚类突变体的小穗密度极显著增加, 小花数和节间 数极显著减少，但小穗数没有明显变化(图 5)。在 5 个亚 类中, 第 1 亚类突变体的小穗数和小花数降幅最大; 第 2 亚类的株高、穗长和平均节间长的降幅和小穗密度的增幅 最大, 第 3 亚类突变体的节间数降幅最大(图 5 和附表 1)。

\section{4 突变农艺性状间的相关分析}

基于 26 个突变体及其诱变亲本的株高等 7 个性状的 二元相关和偏相关分析表明, 株高与穗长、小穗密度和平 均节间长等 4 个性状的二元相关系数达到了显著或极显 著水平, 但只有平均节间长和节间数与株高偏相关; 穗长 与小穗数、小穗密度和平均节间长的二元相关系数达到了 显著或极显著水平, 但只有小穗密度和平均节间长与穗 长存在负偏相关; 小穗数与小穗密度和小花数的二元相 关和偏相关系数均达到了极显著水平; 小穗密度与小花 数和节间数的二元相关系数达到了显著或极显著水平, 但偏相关系数不显著; 虽然平均节间长与节间数的二元 相关系数不显著, 但偏相关系数达到了显著水平 (表 4)。 上述结果表明，26 个突变体的株高与平均节间长和节间 数关联遗传, 其余 4 个性状与植株矮化没有遗传关联性。

\section{3 讨论}

通过诱变亲本和候选突变体连续 2 个年度的株高比 对分析, 作者发现了 26 个矮秆突变体。基于前人报道的 矮秆基因的 12 个特异性标记检测结果表明 26 个矮化材料 至少携带有 2 个矮秆基因标记位点。虽然 mutant 1 、mutant 3 和 mutant 13 等 7 个候选突变体中也检测到了矮秆基因突 变, 但植株偏高, 为 $(33.76 \pm 10.16) \sim(63.99 \pm 2.51) \mathrm{cm}$, 且与 诱变亲本“云麦 53 '的株高没有显著差异。笔者认为这可能 是 2 个年份间的株高波动降低了“ $t$ ”值所致。在 33 个候选 突变体中, 26 个材料明显矮化、且携带不同的矮秆突变基 因, 因此可判定为矮秆突变体。

除株高外, 26 个矮秆突变体还携带穗长缩短、小花密 度增加和节间数减少等不同突变性状。通过分子检测本研 

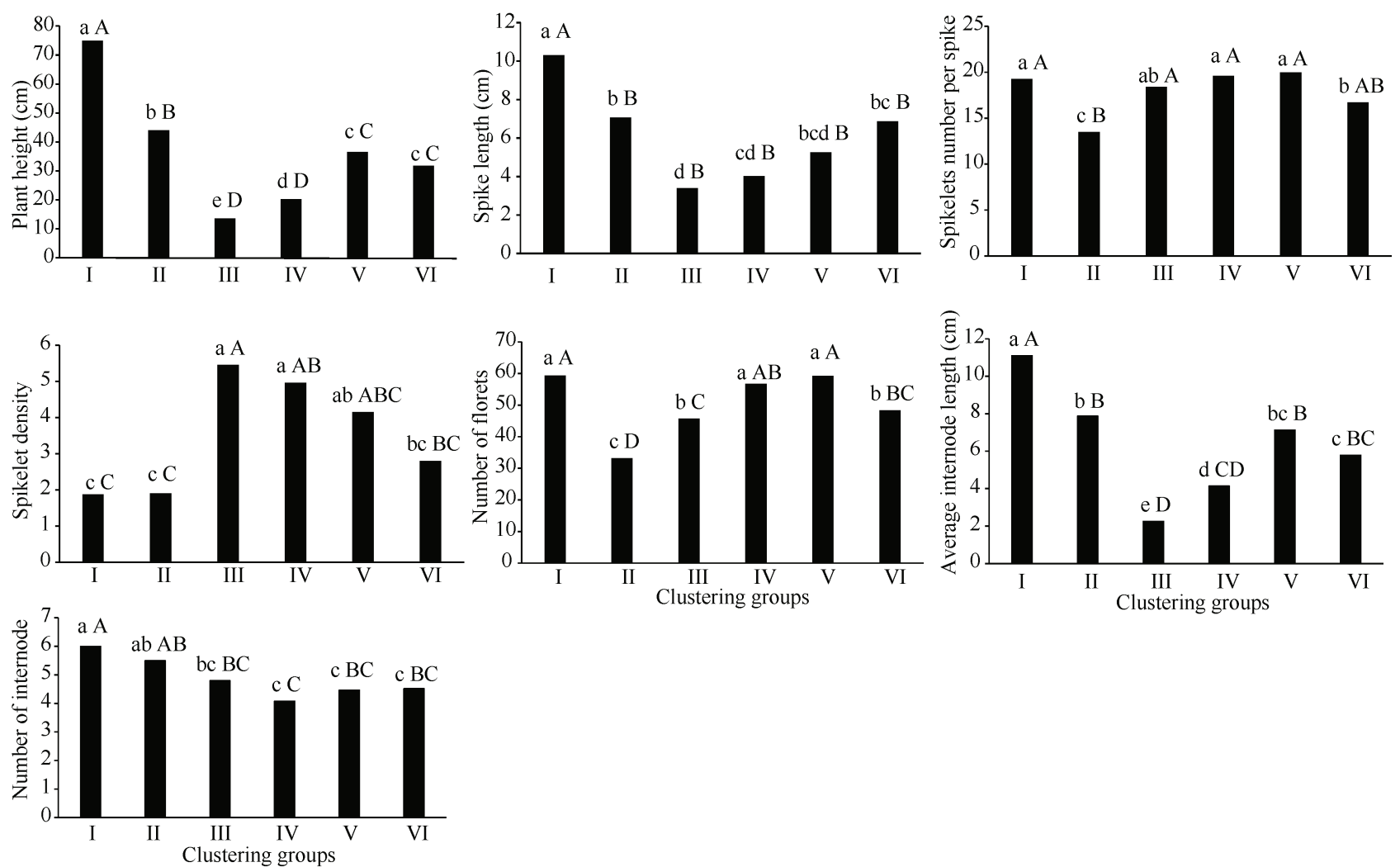

图 5 不同类群材料的农艺性状均值及其差异

Fig. 5 Mean values and differences of agronomic traits in different groups materials

I: ‘云麦 53’类群; II VI: 突变体的第 1 亚类 第 5 亚类。不同小写字母和大写字母分别代表 0.05 和 0.01 显著水平。

I: group 'Yunmai 53'; II-VI: the subclass 1-subclass 5 of mutants. Different lowercase letters and capital letters represent significant differences at the 0.05 and 0.01 probability levels, respectively.

\section{表 4 不同突变性状的二元相关和偏相关系数及其显著性}

Table 4 Binary correlation, partial correlation coefficients and significances among the different mutant traits

\begin{tabular}{|c|c|c|c|c|c|c|c|}
\hline $\begin{array}{l}\text { 性状 } \\
\text { Trait }\end{array}$ & $\begin{array}{c}\text { 株高 } \\
\text { Plant height } \\
(\mathrm{cm}) \\
\end{array}$ & $\begin{array}{c}\text { 穗长 } \\
\text { Spike length } \\
(\mathrm{cm}) \\
\end{array}$ & $\begin{array}{c}\text { 小穗数 } \\
\text { Spikelets number } \\
\text { per spike } \\
\end{array}$ & $\begin{array}{c}\text { 小穗密度 } \\
\text { Spikelet } \\
\text { density } \\
\end{array}$ & $\begin{array}{c}\text { 小花数 } \\
\text { Number of } \\
\text { florets }\end{array}$ & $\begin{array}{c}\text { 平均节间长 } \\
\text { Average internode } \\
\text { length }(\mathrm{cm})\end{array}$ & $\begin{array}{c}\text { 节间数 } \\
\text { Number of } \\
\text { internode }\end{array}$ \\
\hline 株高 Plant height (cm) & & $0.51^{* *}$ & -0.09 & $-0.47^{*}$ & 0.06 & $0.92^{* *}$ & $0.59^{* *}$ \\
\hline 穗长 Spike length $(\mathrm{cm})$ & 0.26 & & $-0.54^{*}$ & $-0.95^{* *}$ & -0.25 & 0.36 & $0.53^{* *}$ \\
\hline 小穗数 Spikelets number per spike & 0.26 & 0.41 & & $0.74^{* *}$ & $0.89^{* *}$ & -0.07 & -0.27 \\
\hline 小穗密度 Spikelet density & -0.11 & $-0.95^{* *}$ & $0.64^{* *}$ & & $0.47^{*}$ & -0.38 & $-0.48^{* *}$ \\
\hline 小花数 Number of florets & -0.32 & 0.13 & $0.78^{* *}$ & -0.09 & & 0.10 & -0.23 \\
\hline 平均节间长 Average internode length (cm) & $0.94^{* *}$ & $-0.43^{*}$ & -0.16 & -0.29 & 0.35 & & 0.35 \\
\hline 节间数 Number of internode & $0.58^{* *}$ & 0.16 & -0.10 & 0.16 & -0.08 & $-0.44^{*}$ & \\
\hline
\end{tabular}

"和"*分别代表 0.05 和 0.01 显著水平。

${ }^{*}$ and ${ }^{* *}$ represent significant differences at the 0.05 and 0.01 probability levels, respectively.

究发现 26 个突变体均携带与‘云麦 53’不同的矮秆基因组 合, 如 mutant 11 携带 Rht 5 和 Rht 12 矮秆基因(可能还携 带其他未被检测的矮秆基因), mutant 2 携带 $R h t-D 1 b$ 、 $R h t 4 、 R h t 5$ 等 9 个矮秆基因。通过推广品种株高与穗长、 小穗数和穗粒数的关联分析，陈亮等 ${ }^{[8-12]}$ 发现矮秆突变基 因 Rht-D 1b、Rht8 和 $R h t 9$ 可增加穗粒数; 王清海等 ${ }^{[13-14]}$ 研究发现 Rht8、Rht10、Rht12、Rht14、Rht16、Rht18 等 矮秆基因的效应不同, Rht10 和 $R h t 12$ 的降秆效应大于
$R h t 8$ 的 ${ }^{[3,5]}$; Baloch 等 ${ }^{[16]}$ 发现 Rht14、Rht16 和 Rht18 可增 加穗长, Rht 5 可减少每穗小穗数。前人研究结果表明小麦 矮秆基因对穗长、小穗数和穗粒数等性状可能具有多效性, 但在不同遗传背景中矮秆基因的遗传效应不同。因此作者 认为是矮秆基因与穗长、小穗密度和小花数等性状的突变 基因及其组合不同导致了 26 个突变体的农艺性状差异。

本研究发现株高与平均节间长和节间数、以及穗长与 小穗数、小穗数与小穗密度、小穗与小花数等性状关联遗 
传, 该结果与前人报道的基本一致 ${ }^{[22-32]}$ 。虽然株高降低后 mutant 7 等 3 个突变体的小穗数和小花数增多(均未达到 显著水平), 其余突变体的穗长缩短、小穗数和小花数减 少、小穗密度增加, 但株高与穗长等 4 个性状的偏相关系 数均未达到显著水平。作者认为该结果可能与本文研究所 用材料大多为相同亲本诱导的矮秆突变体有关, 可能是 矮秆基因及其组合的多效性较低或者矮秆与其他性状的 突变基因位点的连锁强度较低导致了株高与穗长、小穗数 和小穗密度等性状不能关联遗传。通过进一步自交繁育, 可望从 26 份矮秆突变体中䇻选到适合不同育种需求的矮 秆亲本。

通过聚类分析, 作者发现 26 个矮秆突变体可分为农 艺特性不同的 5 个亚类，不同亚类间株高、穗长和小穗数 等农艺性状存在差异, 其中第 2 亚类和第 3 亚类的 mutant 5 、 mutant 23 和 mutant 25 等 5 个突变体株高不到 $20 \mathrm{~cm}$, 均 低于陆燕等的报道 ${ }^{[12,17,33-35]}$ 。虽然株高较低, 但 mutant 11 、 mutant 27 和 mutant 28 等突变体的穗长、小穗数和小花数 没有变化, 且节间数只是导致部分突变体矮化的因素之 一, 因此上述材料可用于小麦矮化育种。除此之外, 26 个 突变体携带的株高、穗长和小穗密度等突变性状基因及其 组合类型不同, 如 mutant 16 携带株高较低 $(13.61 \mathrm{~cm} \pm 0.11 \mathrm{~cm})$, 穗长 $(3.72 \mathrm{~cm} \pm 0.02 \mathrm{~cm})$ 和平均节间长 $(4.88 \mathrm{~cm} \pm 0.62 \mathrm{~cm})$ 较 短, 小穗密度 $(5.37 \pm 0.24)$ 较高的基因组合; mutant 18 具有 植株偏高 $(44.08 \mathrm{~cm} \pm 1.73 \mathrm{~cm})$, 穗长偏长 $(7.08 \mathrm{~cm} \pm 0.48 \mathrm{~cm})$ 小花数较少(33.25 0.25 )的基因组合。基于上述突变体，可 望解析小麦株高及其构成因素、小穗密度和小花数等性状 的遗传机制。

\section{References}

[1] 孙树贵, 李艳丽, 鲁敏, 张军, 王秀娟, 武军, 赵继新, 杨群慧, 刘淑会, 陈新宏. 67 份美国小麦品种矮秆基因的分子标记检测. 麦类作物学报, 2013, 33: 1087-1092.

Sun S G, Li Y L, Lu M, Zhang J, Wang X J, Wu J, Zhao J X, Yang Q H, Liu S H, Chen X H. Distribution of dwarfing genes in 67 American wheat cultivars detected by molecular markers. $J$ Triticeae Crops, 2013, 33: 1087-1092 (in Chinese with English abstract).

[2] 张德强, 宋晓朋, 冯洁, 马文洁, 武炳瑾, 张传量, 崔紫霞, 冯 毅, 孙道杰. 黄淮麦区小麦品种矮秆基因 $R h t-B l b 、 R h t-D l b$ 和 $R h t 8$ 的检测及其对农艺性状的影响. 麦类作物学报, 2016, 36: 975-981.

Zhang D Q, Song X P, Feng J, Ma W J, Wu B J, Zhang C L, Cui Z X, Feng Y, Sun D J. Detection of dwarf genes Rht-Blb, $R h t-D 1 b$ and $R h t 8$ in Huang-Huai Valley winter wheat areas. $J$ Triticeae Crops, 2016, 36: 975-981 (in Chinese with English abstract).

[3] 备凯, 李春游, 敬紮, 陈真真, 王亮明, 王秀娟, 杨群慧, 王中 华, 陈新宏. 部分印度小麦品种矮秆基因的检测及其对部分 性状的影响. 麦类作物学报, 2015, 35: 910-917.

Zan K, Li C Y, Jing F, Chen Z Z, Wang L M, Wang X J, Yang Q $\mathrm{H}$, Wang $\mathrm{Z} \mathrm{H}$, Chen $\mathrm{X} \mathrm{H}$. Detection of dwarfing genes in some
India wheat cultivars and their influences on partial agronomic characteristics. J Triticeae Crops, 2015, 35: 910-917 (in Chinese with English abstract).

[4] 周强, 袁中伟, 欧俊梅, 任勇, 杜小英, 陶军, 李生荣, 刘登才, 四川小麦主要矮秆基因的分子鉴定. 麦类作物学报, 2015, 35: 1624-1630.

Zhou Q, Yuan Z W, Ou J M, Ren Y, Du X Y, Tao J, Li S R, Liu D C. Molecular identification of the main dwarfing genes in wheat varieties in Sichuan. $J$ Triticeae Crops, 2015, 35: 1624-1630 (in Chinese with English abstract).

[5] 朱浩, 牛艳路, 王佩, 全军利, 陈耀锋. 小麦矮秆基因的分子 检测及遗传效应研究. 分子植物育种, 2018, 16: 1855-1861.

Zhu H, Niu Y L, Wang P, Quan J L, Chen Y F. Molecular detection and genetic effect research of dwarf gene in wheat. Mol Plant Breed, 2018, 16: 1855-1861 (in Chinese with English abstract).

[6] 周强, 袁中伟, 张连全, 窂顺腙, 任勇, 陶军, 李生荣, 刘登才. 四倍体小麦地方品种矮蓝麦矮种性状的遗传分析. 作物学报, 2015, 41: 1899-1905.

Zhou Q, Yuan Z W, Zhang L Q, Ning S Z, Ren Y, Tao J, Li S R, Liu D C. Genetic Analysis on dwarfing trait in landrace Ailanmai of Triticum turgidum L. ssp. turgidum. Acta Agron Sin, 2015, 41: 1899-1905 (in Chinese with English abstract).

[7] 钟明志, 魏淑红, 彭正松, 杨在君. 小麦 Rht 矮秆基因研究和 应用综述. 分子植物育种, 2018, 16: 6670-6677.

Zhong M Z, Wei S H, Peng Z S, Yang Z J. A review of the research and application of Rht dwarf genes in wheat. Mol Plant Breed, 2018, 16: 6670-6677 (in Chinese with English abstract).

[8] Emma M J, Rachel E J, Alanna J O, John M M, Michael J G. The impact of the wheat Rht-B1b semi-dwarfing allele on photosynthesis and seed development under field conditions. Front Plant Sci, 2019, 10: 10-51.

[9] 陈亮. 矮秆基因 Rht 12 对小麦重要农艺性状的遗传效应及新矮 秆突变体的篩选. 西北农林科技大学博士学位论文, 陕西杨 凌, 2014. pp 10-19.

Chen L. Genetic Effects of Dwarfing Gene Rht12 on the Important Agronomic Traits of Common Bread Wheat and Screening of New Dwarf Mutants. PhD Dissertation of Northwest A \&F University, Yangling, Shaanxi, China, 2014. pp 10-19 (in Chinese with English abstract).

[10] 唐娜, 逯芳芳, 何蓓如, 胡银岗. 矮秆基因对小麦部分农艺性 状的效应. 西北植物学报, 2010, 30: 41-49.

Tang N, Lu F F, He B R, Hu Y G. Effects of dwarfing genes on some agronomic characteristics of wheat. Acta Bot BorealOccident Sin, 2010, 30: 41-49 (in Chinese with English abstract).

[11] 刘晴, 古佳玉, 赵紫伟, 赵林姝, 郭会君, 谢永盾, 宋希云, 刘 录祥. 小麦矮秆突变体 DC20 的转录组分析. 核农学报, 2019, 33: 1451-1458.

Liu Q, Gu J Y, Zhao Z W, Zhao L S, Guo H J, Xie Y D, Song X Y, Liu L X. RNA-Seq analysis of wheat dwarf mutant DC20. J Nucl Agric Sci, 2019, 33: 1451-1458 (in Chinese with English abstract).

[12] 陆燕, 赵天祥, 刘国祥, 贾继增, 孔秀英. 小麦矮秆圆粒突变 体的鉴定与分析. 植物遗传资源学报, 2014, 15: 160-164. Lu Y, Zhao T X, Liu G X, Jia J Z, Kong X Y. Identification and 
analysis of the dwarf-spherical grain mutant W98. J Plant Genet Resour, 2014, 15: 160-164 (in Chinese with English abstract).

[13] 李杏普, 兰素缺, 张业伦, 张京惠, 冯延茹, 刘书娥, Gale M D, Worland T J. Rht8、Rht10、Rht12 矮秆基因对小麦营养生长和 生殖生长发育的影响. 华北农学报, 2009, 24(增刊 1): 50-53. Li X P, Lan S Q, Zhang Y L, Zhang J H, Feng Y R, Liu S E, Gale $\mathrm{M}$ D, Worland $\mathrm{T}$ J. Effects of Rht8, Rht10 and Rht12 semi-dwarfing and dwarfing genes on wheat vegetative and reproductive development. Acta Agric Boreali-Sin, 2009, 24(S1): 50-53 (in Chinese with English abstract).

[14] 王清海, 杨在君, 魏淑红, 廖明莉, 苏瑾, 杨宇凤, 杨会, 王育 伟, 彭正松. 四倍体小麦矮秆基因的赤霉素敏感性及对农艺 性状的影响. 华北农学报, 2015, 30(2): 132-139.

Wang Q H, Yang Z J, Wei S H, Liao M L, Su J, Yang Y F, Yang H, Wang Y W, Peng Z S. Gibberellin sensitivity of the Rht genes in tetraploid wheat and effects on agronomic traits. Acta Agric Boreali-Sin, 2015, 30(2): 132-139 (in Chinese with English abstract).

[15] Baloch G A, Cui L, Hu Y G. The effects of dwarfing gene Rht-8 on plant height and other agronomic traits in common wheat. Agric Res Arid Areas, 2014, 32: 245-252.

[16] Sun L H, Yang W L, Li Y F, Shan Q Q, Ye X B, Wang D Z, Yu K, Lu W W, Xin P Y, Pei Z, Guo X L, Liu D C, Sun J Z, Zhan K H, Chu J F, Zhang A M. A wheat dominant dwarfing line with Rht12, which reduces stem cell length and affects gibberellic acid synthesis, is a 5AL terminal deletion line. Plant J, 2019, 97: 887-900.

[17] 卢媛, 崔超凡, 胡平, 陈佩度, 沈雪芳, 韩晴, 王义发, 邢莉萍, 曹爱忠. 矮秆基因 Rht_NM9 在小麦株高建成中对内源激素含 量的影响. 作物学报, 2017, 43: 1272-1279.

Lu Y, Cui C F, Hu P, Chen P D, Shen X F, Han Q, Wang Y F, Xing L P, Cao A Z. Effects of dwarf gene Rht_NM9 on contents of endogenous hormone regulating plant height of common wheat. Acta Agron Sin, 2017, 43: 1272-1279 (in Chinese with English abstract).

[18] Ellis M H, Rebetzke G J, Azanza F, Richards R A, Spielmeyer W. Molecular mapping of gibberellin responsive dwarfing genes in bread wheat. Theor Appl Genet, 2005, 111: 423-430.

[19] Ellis M H, Spielmeyer W, Rebetzke G J, Richards R A. Perfect markers for the and dwarfing genes in wheat. Theor Appl Genet, 2002, 105: 1038-1042.

[20] Watanabe N. Genetic collection and development of near-isogenic lines in durum wheat. Czech J Genet Plant, 2008, 12: 636-643.

[21] Korzun V, Roeder M S, Ganal M W, Worland A J, Law C N. Genetic analysis of the dwarfing gene in wheat: Part I. molecular mapping of on the short arm of chromosome 2D of bread wheat. Theor Appl Genet, 1998, 96: 1104-1109.

[22] 贾影影. 小麦矮秆基因 Rht 13 与 $R h t-D 1 b$ 互作研究及 $R h t 13$ 分 子标记开发. 宁夏大学硕士学位论文, 宁夏银川, 2016. pp $22-26$.

Jia Y Y. Study on Interaction of Dwarfing Gene Rht13 with Rht-D1b and Development Molecular Markers for Rht13 in Wheat. MS Thesis of Ningxia University, Yinchuan, Ningxia, China, 2016. pp 22-26 (in Chinese with English abstract).

[23] 吴同彦, 谢令琴, 杨学举, 张彩英, 陈荣芬. 小麦株高构成因
素与产量及其他性状相关性的研究. 河北农业大学学报, 2002, 25(3): 10-12.

Wu T Y, Xie L Q, Yang X J, Zhang C Y, Chen R F. Study on the correlations between the components of the plant height and the yield and other traits of wheat. J Agric Univ Hebei, 2002, 25(3): 10-12(in Chinese with English abstract).

[24] 闵东红, 王辉, 孟超敏, 翟耀锋, 李学军, 曹宁, 于新智. 不同 株高小麦品种抗倒伏性与其亚性状及产量相关性研究. 麦类 作物学报, 2001, 21(4): 76-79.

Min D H, Wang H, Meng C M, Zhai Y F, Li X J, Cao N, Yu X Z. Studies on the lodging resistance with its subtraits of different height wheat varieties and correlation between plant height and yield. J Triticeae Crops, 2001, 21(4): 76-79 (in Chinese with English abstract).

[25] 赵万春, 王红. 小麦株高及其构成因素的遗传和相关性研究. 麦类作物学报, 2003, 23(4): 28-31.

Zhao W C, Wang H. Genetic and correlation study on plant height and its components in wheat. J Triticeae Crops, 2003, 23(4): 28-31 (in Chinese with English abstract).

[26] 张坤普, 徐宪斌, 田纪春. 小麦籽粒产量及穗部相关性状的 QTL 定位. 作物学报, 2009, 35: 270-278.

Zhang K P, Xu X B, Tian J C. QTL Mapping for grain yield and spike related traits in common wheat. Acta Agron Sin, 2009, 35: 270-278 (in Chinese with English abstract).

[27] 张晶, 张定一, 王姣爱, 党建友. 小麦单株有效分菜数与农艺 性状的相关性研究. 山西农业科学, 2009, 37(6): 17-19.

Zhang J, Zhang D Y, Wang J A, Dang J Y. The dependence study of the effective tillers per plant and agronomic characters in wheat. J Shanxi Agric Sci, 2009, 37(6): 17-19 (in Chinese with English abstract).

[28] 卢翔, 张锦鹏, 王化俊, 杨欣明, 李秀全, 李立会. 小麦-冰草 衍生后代 3558-2 穗部相关性状的遗传分析和 QTL 定位. 植物 遗传资源学报, 2011, 12: 86-91.

Lu X, Zhang J P, Wang H J, Yang X M, Li X Q, Li L H. Genetic analysis and QTL mapping of wheat spike traits in a derivative line 3558-2 from wheat $\times$ Agropyron cristatum offspring. J Plant Genet Resour, 2011, 12: 86-91 (in Chinese with English abstract).

[29] 张祥池, 郝燕冉, 魏凡, 张志锋, 魏佳佳, 付帅, 安浩军, 王睿 辉. 小麦矮秆突变体矮秆基因的遗传分析. 河北农业大学学 报, 2017, 40(2): 5-10.

Zhang X C, Hao Y R, Wei F, Zhang Z F, Wei J J, Fu S, An H J, Wang R H. Characterization of the Rht gene in one dwarfing mutant of wheat (Triticum aestivum L.). J Agric Univ Hebei, 2017, 40(2): 5-10 (in Chinese with English abstract).

[30] 冯洁, 许小宛, 李小东, 张传量, 崔紫霞, 冯毅, 孙道杰. 黄淮 麦区小麦品种和 CIMMYT 材料的矮秆基因型及其对株高和胚 芽鞘的影响. 麦类作物学报, 2018, 38: 668-673.

Feng J, Xu X W, Li X D, Zhang C L, Cui Z X, Feng Y, Sun D J. Dwarf genotype of wheat from Huang-Huai river wheat area and CIMMYT and their effects on plant height and coleoptile length. $J$ Triticeae Crops, 2018, 38: 668-673 (in Chinese with English abstract).

[31] 吴儒刚, 裴艳婷, 张超, 范业泉, 靳义荣, 刘鹏, 贾德新, 戴忠 民. 基于盐胁迫的小麦农艺性状多样性分析及评价. 麦类作 物学报, 2019, 39: 1029-1037. 
Wu R G, Pei Y T, Zhang C, Fan Y Q, Jin Y R, Liu P, Jia D X, Dai $\mathrm{Z} \mathrm{M}$. Analysis and evaluation of agronomic character diversity of wheat based on salt stress. J Triticeae Crops, 2019, 39: 1029-1037 (in Chinese with English abstract).

[32] 林旭群, 李韵芳, 鲁璐, 张春艳, 廖金花, 李莉蓉, 吴瑜. 小麦 矮秆基因 $R h t-B 1 b 、 R h t-D 1 b 、 R h t 8$ 的检测及其对株高的影响. 应用与环境生物学报, 2014, 20: 80-86.

Lin X Q, Li Y F, Lu L, Zhang C Y, Liao J H, Li L R, Wu Y. Identification of wheat dwarfing genes Rht-B1b, Rht-Dlb, Rht8 and their effects on plant height. Chin J Appl Environ Biol, 2014, 20: 80-86 (in Chinese with English abstract).

[33] 王鍂, 马荣雪, 杨阳, 王丹峰, 殷慧娟, 王洪刚. 小麦矮秆种 质 SN224 的鉴定及农艺性状 QTL 分析. 作物学报, 2016, 42: 1134-1142.

Wang X, Ma Y X, Yang Y, Wang D F, Yin H J, Wang H G. Identification of dwarfing wheat germplasm SN224 and analysis of
QTLs for its agronomic characters. Acta Agron Sin, 2016, 42: 1134-1142 (in Chinese with English abstract).

[34] 杨秋平, 杨阳, 王金錪, 马芗雪, 张明, 王洪刚. 小麦矮秆种质 山农 11069-5 矮秆基因的遗传分析及分子定位. 分子植物育种, 2015, 13: 71-76.

Yang Q P, Yang Y, Wang X, Ma Y X, Zhang M, Wang H G. Genetic analysis and molecular mapping of the gene in dwarf germplasm Shannong 11069-5. Mol Plant Breed, 2015, 13: 71-76 (in Chinese with English abstract).

[35] 张明, 吴瑕, 张一铎, 张超, 牛祖彪, 崔淑佳, 杨秋平, 王洪刚. 小麦矮秆种质系山农 342-9 矮秆基因的分子标记定位. 山东农 业科学, 2014, 46(7): 7-10.

Zhang M, Wu X, Zhang Y D, Zhang C, Niu Z B, Cui S J, Yang Q P, Wang H G. Molecular mapping of Rht Gene in wheat dwarfing germplasm line Shannong 342-9. Shandong Agric Sci, 2014, 46(7): 7-10 (in Chinese with English abstract).

附表 1 '云麦 53' 类群和 5 个矮秆突变体亚类的方差分析结果

Table S1 ANOVA result of 'Yunmai 53' and 5 subclasses dwarf mutants

\begin{tabular}{|c|c|c|c|c|c|}
\hline $\begin{array}{l}\text { 性状 } \\
\text { Traits }\end{array}$ & $\begin{array}{c}\text { 变异来源 } \\
\text { SOV }\end{array}$ & $\begin{array}{c}\text { 平方和 } \\
\mathrm{SS} \\
\end{array}$ & $\begin{array}{c}\text { 自由度 } \\
d f\end{array}$ & $\begin{array}{c}\text { 均方 } \\
\text { MS }\end{array}$ & $\begin{array}{c}F \text { 值 } \\
F \text {-value }\end{array}$ \\
\hline \multirow[t]{2}{*}{ 株高 Plant height (cm) } & 组间 Between groups & $11,981.952$ & 5 & 2396.390 & $37.615^{* *}$ \\
\hline & 组内 Within group & 4077.292 & 64 & 63.708 & \\
\hline \multirow[t]{2}{*}{ 穗长 Spike length (cm) } & 组间 Between groups & 119.531 & 5 & 23.906 & $4.603^{* *}$ \\
\hline & 组内 Within group & 332.417 & 64 & 5.194 & \\
\hline \multirow[t]{2}{*}{ 小穗数 Spikelets number per spike } & 组间 Between groups & 234.415 & 5 & 46.883 & $10.229^{* *}$ \\
\hline & 组内 Within group & 293.328 & 64 & 4.583 & \\
\hline \multirow[t]{2}{*}{ 小穗密度 Spikelet density } & 组间 Between groups & 48.716 & 5 & 9.743 & $6.920^{* *}$ \\
\hline & 组内 Within group & 90.110 & 64 & 1.408 & \\
\hline \multirow[t]{2}{*}{ 小花数 Number of florets } & 组间 Between groups & 3126.921 & 5 & 625.384 & $11.289^{* *}$ \\
\hline & 组内 Within group & 3545.316 & 64 & 55.396 & \\
\hline \multirow[t]{2}{*}{ 平均节间长 Average internode length $(\mathrm{cm})$} & 组间 Between groups & 190.020 & 5 & 38.004 & $13.307^{* *}$ \\
\hline & 组内 Within group & 182.776 & 64 & 2.865 & \\
\hline \multirow[t]{2}{*}{ 节间数 Number of Internode } & 组间 Between groups & 14.628 & 5 & 2.926 & $7.604^{* *}$ \\
\hline & 组内 Within group & 24.623 & 64 & 0.385 & \\
\hline
\end{tabular}

"表示在 0.01 水平差异显著。

${ }^{* *}$ mean significant difference at the 0.01 probability level. 